«Ça va être un viol » : Formes et fonctions de l'obscénité langagière dans les joutes verbales de rap

\title{
Cyril Vettorato
}

\section{(2) OpenEdition} Journals

Édition électronique

URL : https://journals.openedition.org/clo/1492

DOI : $10.4000 /$ clo. 1492

ISSN : 2266-1816

Éditeur

INALCO

\section{Édition imprimée}

Date de publication : 1 janvier 2012

Pagination : 115-140

ISBN : 978-2-85831-206-1

ISSN : 0396-891X

\section{Référence électronique}

Cyril Vettorato, « « Ça va être un viol » : Formes et fonctions de l'obscénité langagière dans les joutes verbales de rap », Cahiers de littérature orale [En ligne], 71 | 2012, mis en ligne le 27 avril 2014, consulté le 01 juillet 2021. URL : http://journals.openedition.org/clo/1492 ; DOI : https://doi.org/10.4000/clo. 1492

Ce document a été généré automatiquement le 1 juillet 2021.

\section{(c) (i) (9)}

Cahiers de littérature orale est mis à disposition selon les termes de la Licence Creative Commons Attribution - Pas d'Utilisation Commerciale 4.0 International. 


\title{
«Ça va être un viol »: Formes et fonctions de l'obscénité langagière dans les joutes verbales de rap
}

\author{
Cyril Vettorato
}

1 La pratique des freestyle battles chers aux passionnés de hip-hop est caractéristique du statut ambivalent des pratiques poétiques orales issues des cultures dites « urbaines ». Entre virtualité et actualisation, elles font apparaitre avec éclat un ensemble de symboles et de valeurs extrêmement bien maîtrisés par leurs acteurs, maîtrise qui est sans commune mesure avec la fréquence à laquelle ces pratiques sont réellement exercées. Le battle, un tournoi opposant deux rappeurs qui se lancent au visage des couplets improvisés bardés d'insultes et de moqueries, est toujours relativement rare en France. Néanmoins, lors du battle, chacun des acteurs semble connaître sa place et agir en fonction d'elle. L'apparition en 2010 de la première ligue de battle française, basée à Paris et dénommée Rap Contenders, en fournit un exemple frappant. Le premier tournoi organisé par cette ligue a eu lieu le 11 décembre 2010 dans le dix-neuvième arrondissement de la capitale et a réuni douze rappeurs français ; c'est ce tournoi qui retiendra particulièrement notre attention. L'événement, qui a été filmé dans son intégralité, est exceptionnel, à défaut d'être le premier du genre. Et pourtant, les règles du jeu sont parfaitement maîtrisées par tous, créant les conditions d'une mécanique ludique fonctionnelle, prête à se mettre en marche autant de fois que possible. Nous sommes ici au cœur de la génération virtuelle, qui s'est familiarisée avec le hip-hop et ses codes sur Internet et à la télévision. Elle a autant, sinon plus, fréquenté les tournois de battles dans le film à succès 8 Mile - et sur les sites de partage de vidéos en ligne - que dans le vacarme des salles de concerts. Peu importe au final que le jeu soit pratiqué pour la première fois : les mots, les symboles, les postures et les valeurs qu'il engage le dépassent de loin, même si elles ont besoin de ces tumultueux moments d'actualisation pour exister.

2 Deux éléments, plus directement liés au contenu des couplets improvisés, frappent immédiatement les oreilles lorsque l'on visionne les quatre-vingt-sept minutes de vidéo 
de ce premier tournoi parisien, qui a opposé douze rappeurs en six battles de trois rounds chacun. Il s'agit tout d'abord du caractère profondément conventionnel des répliques. Alors que le rap pratiqué par des artistes musicaux peut traiter de thèmes très variés, le rap improvisé des battles se contente de quelques idées récurrentes, opérant davantage sur le mode de la variation sur le même thème. Le rappeur qui a la parole dispose d'une minute - parfois jusqu'à trente secondes de plus, en fonction du bon vouloir du juge - pour couvrir son adversaire d'injures tournées de manière à provoquer l'hilarité du public. Le plus souvent, ces injures ne sont que des variations sur la virilité du locuteur et l'absence de virilité de son adversaire. La question sexuelle, et ce sera là notre seconde remarque introductive, imprègne la majorité des rimes des rappeurs de battle. Il existe un certain nombre de thèmes secondaires comme la laideur de l'adversaire ou l'échec de sa carrière musicale, mais tout revient très vite à une évocation plus ou moins indirecte de rapports sexuels, en des termes témoignant de la prégnance d'un imaginaire pornographique. N'oublions pas que l'un des ancêtres de cette forme de joute est le jeu africain américain ${ }^{1}$ des Dirty Dozens, qui consiste à insulter la mère de son opposant à travers des répliques souvent scatologiques et/ou pornographiques. La génitrice des adversaires est donc logiquement traînée dans la boue, ainsi que, parfois, sa sœur et sa petite amie. Mais si les insultes envers les proches féminines de l'adversaire sont fréquentes, l'observation précise des battles nous enseigne qu'elles ne sont pas le seul, ni même le principal motif de mobilisation d'une imagerie pornographique et scatologique.

3 Au cours du tournoi Rap Contenders de décembre 2010, nous avons relevé cinquante-six évocations de rapports sexuels par les participants, lors de leurs couplets improvisés. Sur tous ces cas, seuls quatorze renvoyaient à un rapport hétérosexuel, tandis que les quarante-deux autres avaient pour objet un rapport homosexuel entre hommes. Cette proportion d'un à trois témoigne de l'omniprésence, au sein des battles de rap, de l'imaginaire du rapport charnel homosexuel. Au cours de ces six battles, aucun participant n'a proposé de couplets dépourvus de références à cette thématique ; sur trente-six freestyles (couplets improvisés) d'une minute à une minute trente, on dénombre seize références à la sexualité des proches féminines de l'adversaire, et pas moins de soixante-trois références à l'homosexualité supposée de ce dernier. Dans l'imaginaire de la virilité qui est valorisé par ces rappeurs, le fait de recourir à cette attaque est à l'évidence un moyen de disqualifier son concurrent. Il ne s'agit jamais d'affirmer littéralement que son adversaire est homosexuel, mais bien de recourir aux connotations négatives associées à l'homosexualité au sein du système commun de valeurs pour construire des punchlines ${ }^{2}$. En outre, la réplique portant sur des actes sexuels entre hommes permet d'effectuer une double transgression, celle du tabou langagier et celle relative au tabou de l'homophobie.

4 Nous tenterons ici de cerner les enjeux des recours à l'obscénité sexuelle et scatologique dans les battles, obscénité qui renvoie le plus souvent au rapport homosexuel masculin. Le rappeur Gaïden, vainqueur du troisième battle du tournoi qui l'oppose à Srin Po, lance ainsi au public hilare peu après le début de son couplet : «Eh attendez c'est pas fini ça va être un viol» $(2: 19)$; et de poursuivre avec une série d'évocations explicites de rapports sexuels entre lui et son adversaire. Nous sommes en présence d'un groupe d'acteurs qui associent des valeurs très négatives à ce type de rapports sexuels, mais qui le mobilisent de manière presque obsessionnelle et pour le moins ambiguë. S'agit-il uniquement d'une quête de transgression verbale et morale, porteuse de valeurs contre-culturelles? Les «mots sales» sont-ils les vecteurs d'une 
forme d'identité de groupe, perceptible à travers les codes de la joute? Les images pornographiques et scatologiques, souvent liées à un imaginaire homophobe, finissentelles par constituer une contre-norme dans laquelle se reconnaissent les acteurs, et si oui comment? Il faudra surtout nous demander à quel niveau fonctionnent les mécanismes d'évaluation des "vannes » et des punchlines, ces "rimes coup de poing" chargées de détruire l'adversaire sur la scène du langage. Le seul recours aux valeurs positives ou négatives attachées à une image suffit-il à assurer l'efficacité d'un effet? Afin d'apporter des éléments de réponse à ces questions, nous commencerons par quelques rappels historiques avant d'évoquer le fonctionnement concret des battles de notre corpus, en nous attachant spécifiquement au rôle qui est dévolu aux images pornographiques et scatologiques; nous proposerons ensuite une lecture de ce rôle, en particulier dans son rapport à l'efficacité discursive. Ces étapes pourront servir de base à une interprétation des phénomènes de transgression langagière dans les joutes verbales de hip-hop, dont l'imaginaire sexuel volontiers brutal, misogyne et homophobe n'a pas manqué d'inspirer nombre de commentateurs extérieurs.

\section{De la transgression à l'agression}

Dans sa chanson «Le Duc de Boulogne », qui date de 2006, le rappeur Booba résume en quelques mots le rapport qu'entretient le hip-hop à la transgression langagière. Après un très raffiné « Baby, fais-moi la bise, puis suce-moi la bite », il reconnaît : « OK je suis vulgaire, les bourges en chopent des ulcères, quand ils écoutent ce qui sort de ma bouche c'est le son dangereux du ter-ter ». Cet aparté méta-poétique en dit long sur ce qui est en jeu ici. Le «ter-ter ", c'est le quartier, lieu symbolique de l'authenticité et de la rugosité du quotidien autour duquel est constituée la communauté projective du rap. Quand bien même un artiste ne vivrait plus dans un environnement social répondant à ces critères, voire n'y aurait jamais vécu, ce lieu demeure en toile de fond de sa pratique. Le «ter-ter » est le mètre-étalon du rap, et les «bourges» que Booba avoue ici chercher à choquer représentent la société française dans son ensemble, conçue comme policée et respectable. Nulle critique sociale ou économique d'inspiration marxiste dans cette référence aux "bourgeois", mais une valorisation de la transgression en tant que telle. Il n'est pas question de proposer un modèle alternatif et utopique de société, mais de s'aménager dans les marges un lieu à soi, marqué par le nihilisme, le matérialisme et la valorisation des codes du groupe. Dans ce cadre, évocations scatologiques et pornographiques, souvent mâtinées de misogynie et d'homophobie, participent d'une même image de soi comme marginaux rejetés par la société et rejetant celle-ci en retour. Les "mots sales" sont portés comme les étendards de ce rapport particulier au monde social.

Lorsque le hip-hop apparaît dans les années 1970 aux États-Unis, cette dimension transgressive est d'ores et déjà présente, tout comme le fonctionnement agonistique observable au sein des battles. La pratique du rap donne rapidement lieu à des formes de joutes informelles, avant de s'organiser des années plus tard sous la forme de tournois comme le Scribble Jam de Cincinnati. L'inspiration des premiers rappeurs se nourrit tout autant des diatribes poétiques virulentes proférées durant les années du «Mouvement pour les Droits civiques » des années 1960 et 1970 par Gil Scott-Heron, les Last Poets et Amiri Baraka, que de formes de jeux sur les mots pratiqués au quotidien dans les quartiers populaires. Ces pratiques orales proviennent aussi bien des États- 
Unis que de la Caraïbe, où ont vu le jour certains des inventeurs du mouvement : outre le jeu des Dozens déjà mentionné, les premiers rappeurs s'inspirent du toast jamaïcain, lui-même peu avare en plaisanteries scatologiques ou sexuelles. Béthune $(2003,137)$ a souligné combien «À sa façon, le rap réactualise de manière explicite un vif penchant pour la poétique de l'obscène, propre à la tradition afro-américaine ». Cette intuition généalogique est confirmée par les linguistes et sociolinguistes spécialisés dans le parler des ghettos noirs américains comme Abrahams (1970, 75). Pour Smitherman également $(1977,62)$, on perçoit dans les jeux langagiers du hip-hop la nette influence de la façon de parler des Africains Américains, qui est «enracinée dans l'expérience culturelle des Noirs, dont la sémantique dépend non seulement du contexte linguistique immédiat, mais aussi du contexte social et historique $»^{3}$. Le traitement ludique des mots, et en particulier des mots interdits, trouve son origine dans cette éthique du langage. C'est de là que proviennent les jeux de désémantisation et de multiplication des significations des mots que l'on peut observer dans le rap. En anglais de la rue, shit, motherfucker ou encore bitch se trouvent détachés de leur sens référentiel initial pour emprunter des sens différents selon les contextes. Le hip-hop voit dans le parler de la rue le garant de sa propre authenticité, puisant dans ses ressources pour se renouveler en permanence. Pour être le meilleur rappeur possible, il convient de savoir employer les termes argotiques les plus récents, comme si l'on était branché en permanence sur l'énergie de la rue. L'univers figural mobilisé est celui du groupe de pairs, en particulier du groupe de pairs jeune et masculin des quartiers populaires, dont les membres passeraient le plus clair de leur temps à plaisanter, à se taquiner et à parler de sexe de manière outrageuse.

7 Nous insistons sur la dimension imaginaire de cette localité du rap, car elle ne doit en aucun cas être confondue avec ses lieux véritables. Comme nous l'avons souligné en introduction, le hip-hop comme phénomène culturel se situe à la jonction du virtuel et de l'effectif. Le moment du battle actualise des valeurs et des images partagées par les acteurs, qui se sont en grande partie familiarisés avec elles par le truchement de l'imaginaire. Certains rappeurs vivent réellement dans les grands ensembles des banlieues françaises, ou y ont vécu; d'autres n'y ont jamais posé le pied, si ce n'est indirectement, en écoutant la radio Skyrock, les disques de NTM, I AM ou Booba, ou encore des enregistrements vidéo de performance mis en ligne sur des sites web de partage. La "communauté hip-hop " n'est pas réductible à un lieu ou à un groupe sociologique réel, même si elle porte en elle un imaginaire profondément enraciné. Pour le dire autrement, le "quartier », le «ter-ter» est moins à comprendre dans ce cas comme un lieu réel que comme une métaphore. Certains rappeurs, y compris parmi les participants du Rap Contenders ne sont aucunement issus des couches populaires de la société, mais ils se reconnaissent dans l'univers du hip-hop et s'identifient à lui. Le crew est le groupe d'amis dont fait partie le rappeur, et reproduit le schéma du " groupe de pairs " urbain; pourtant, le crew peut réunir des personnes issues de lieux et d'horizons très différents, qui se sont rencontrés dans les lieux réels du hip-hop, comme les salles de concert, les MJC (Maisons des Jeunes et de la Culture), les disquaires spécialisés ou les festivals, voire sur Internet. Par exemple, parmi les douze participants du premier tournoi Rap Contenders, quatre appartiennent au crew nommé «L'Entourage » (Alpha Wann, Deen Burbigo, Eff Gee et Nekfeu), alors qu'ils viennent de quartiers différents - le quatorzième arrondissement de Paris pour Alpha Wann, Aubervilliers (93) pour Deen Burbigo, le dix-neuvième arrondissement pour Eff Gee et le quinzième pour Nekfeu. De manière générale, les participants du battle ne présentent 
pas de "profil type ». Certains sont des adolescents, d'autres ont la trentaine bien entamée; certains sont d'origine africaine ou maghrébine, d'autres non. Sur douze rappeurs, six viennent de Paris (Alpha Wann, Donny S, Eff Gee, Gaïden, Nekfeu et Srin Po), y compris d'arrondissements réputés plutôt huppés (le quatorzième et le quinzième), et six de la banlieue parisienne (Seine-Saint-Denis pour Deen Burbigo, Logik Konstantine et Pen, Val-d'Oise pour Blackapar et Lunik, Yvelines pour TZN).

8 Au-delà des considérations sociologiques, le «quartier » peut bien être qualifié de métaphore dans la mesure où il trouve son sens par rapport à un ensemble de valeurs et d'images connexes, formant une sorte de réseau de significations. Il ne s'agit jamais uniquement d'une référence à un lieu réel, mais toujours à la fois d'une sorte de symbole central au sein d'un système de valeurs. Comme l'écrit Bazin (1995, 92), «Les expressions du hip-hop ne dessinent pas un espace géographique, mais existentiel. » Le "quartier ", le "ter-ter", le "tié-car » et le crew sont autant de signifiants qui ne peuvent être compris qu'une fois replacés dans ce réseau complexe. Cette imbrication de symboles nous intéresse tout spécialement ici car la joute elle-même constitue une sorte de mise en scène ritualisée de ces symboles et de ces valeurs. L'événement poétique joue un rôle important dans la mesure où il est le lieu d'élection d'acteurs culturels singuliers, chargés par le groupe de faire advenir les codes du hip-hop de manière nouvelle et particulièrement frappante. Le public, qui se manifeste bruyamment au cours de la performance, n'est pas qu'un simple observateur : c'est en fonction de ses réactions que le rappeur sera reconnu ou non vainqueur, même quand un système de juges est mis en place. Le MC (Maître de Cérémonie, autre nom des rappeurs) qui aura su "tout déchirer ", "mettre le feu», sera déclaré vainqueur. Il devient alors une sorte de "héros culturel », ce qui accroît son prestige au sein de la communauté du hip-hop. Les enregistrements vidéo des battles sont d'ailleurs mis en ligne et abondamment commentés par les passionnés en quête d'un nouveau poulain. L'accession à ce statut prestigieux nécessite la reconnaissance de deux qualités par les pairs: la maîtrise parfaite d'un ensemble de techniques poétiques et prosodiques, manifestées dans une performance originale, et l'exemplification d'un ensemble de valeurs communes. Ces deux niveaux ne doivent pas nécessairement être compris comme séparés; à bien des égards, ils se manifestent l'un par l'autre. Le sujet s'y affirme en étant original, en affirmant son propre style: reprendre les mots ou les traits stylistiques d'un autre est rédhibitoire. Pour ridiculiser son adversaire Deen Burbigo, Blackapar lui lance par exemple ici lors du battle Rap Contenders qui les oppose : "T'as volé le flow à Fabe » $(5: 01)$, sous-entendant qu'il n'a pas de style distinct, mais se contente d'imiter ce rappeur parisien légendaire des années 1990. Mais cette originalité passe toujours par la mise en évidence d'une maîtrise et d'un respect de certaines valeurs reconnues par tous les acteurs. La performance poétique est le surgissement symbolique des valeurs du groupe.

9 Pour nous en convaincre, nous pouvons nous arrêter un instant sur le déroulement du tournoi. Le Rap Contenders de décembre 2010 a réuni douze rappeurs dans une salle de taille moyenne du dix-neuvième arrondissement parisien dans laquelle se pressait un public nombreux et très réactif. Il n'existe pas de scène proprement dite dans cette salle, mais les spectateurs forment un cypher dans la salle, c'est-à-dire qu'ils entourent les participants en leur laissant un espace similaire à une petite scène. Par leur style vestimentaire et par leur promptitude à répondre aux références précises à l'univers mobilisé par les rappeurs, ces membres du public exemplifient la figure du passionné de hip-hop. Presque tous sont de sexe masculin, semblent avoir entre dix-huit et 
quarante ans environ, et portent des casquettes, des pantalons larges et des chaussures de sport. En plus de ce juge pluriel et bruyant, un jury a été désigné pour décider du vainqueur de chaque battle. Il s'agit d'un certain nombre d'hommes reconnus comme des spécialistes de la pratique du freestyle, le plus souvent parce qu'ils s'y adonnent euxmêmes. Dans certains cas, le vainqueur est désigné en fonction des réactions du public, évaluées par un arbitre unique ou mesurées par un système d'applaudimètre. Dans d'autres, un jury est constitué afin d'éviter de privilégier les candidats venus accompagnés d'un groupe d'amis complaisants - ce qui risque de parasiter le fonctionnement du jugement par des pairs en fonction de la qualité de la performance. Les jurés se trouvent au cœur du public, et s'isolent après la fin de la performance pour rendre leur verdict. Ensuite, les deux concurrents sont rappelés dans la salle, et le vainqueur est désigné et acclamé. Deux arbitres sont chargés de superviser le déroulement des battles. Dans le cas étudié, il s'agit de Stunner et Donny S, un rappeur parisien membre du crew Yellow Stone qui participe au sixième combat en tant que concurrent. Les deux arbitres souhaitent la bienvenue au public, puis présentent les deux personnes opposées dans le battle. L'un d'entre eux tire à pile ou face pour décider lequel des deux concurrents aura le droit de choisir s'il exécutera son couplet en premier; puis il donne le coup d'envoi du combat, vérifiant sur un chronomètre la durée de chaque tour de parole.

Dans le Rap Contenders, plusieurs choix spécifiques ont été opérés par les organisateurs. Le tour de parole n'a pas de durée strictement définie, dans la mesure où les couplets sont proférés a capella. Dans la plupart des tournois de battles organisés aux États-Unis, un morceau instrumental accompagne la performance, forçant les concurrents à se tenir à une durée limitée. Le plus souvent, il s'agit de morceaux classiques du hip-hop, sur lesquels les rappeurs doivent improviser un nombre donné de mesures. Ce système contraint les Maîtres de Cérémonie à imaginer des rimes et des plaisanteries très rapidement, ce qui nécessite une maîtrise technique importante. Il faut impérativement que chaque mesure rythmique s'achève sur une rime: manquer une seule de ces rimes est considéré comme un signe de faiblesse. Même si les morceaux choisis sont rarement très rapides, cela force l'esprit à enchaîner les idées en quelques secondes seulement, préparant déjà le vers suivant avant même que celui que l'on est en train de prononcer n'ait atteint les oreilles du public. En France, la rareté des tournois de battles fait que les rappeurs ne sont pas aussi entraînés que leurs homologues américains ou britanniques. Alors que dans un tournoi comme le Scribble Jam, l'improvisation sur fond instrumental est la règle, le Rap Contenders laisse aux rappeurs la liberté de prononcer leurs vers à la vitesse qu'ils souhaitent, et leur reconnaît le droit d'écrire leurs couplets à l'avance. Dans un battle en général, préparer son couplet en amont est rédhibitoire. Parmi les passionnés de ces tournois, les interminables discussions à propos d'oppositions ayant eu lieu portent souvent sur ce point : lorsque des vers semblent peu spontanés, certains affirment qu'ils ont été écrits à l'avance, ce qui disqualifie le concurrent qui les a prononcés. Ici, à l'inverse, il est ouvertement reconnu que les couplets sont écrits et mémorisés à l'avance, même si certains vers sont improvisés par les concurrents suite à des trous de mémoire comme dans le cas d'Alpha Wann. Cette flexibilité augmente le rôle des arbitres, chargés de déclarer la fin du tour de parole en effectuant un geste vertical du bras. Les rappeurs disposent d'une minute à une minute trente, et les arbitres se montrent plutôt conciliants, accordant volontiers des secondes supplémentaires en cas d'oublis ou d'hésitations. 
11 La joute poétique est en tous points présentée comme une sorte de combat sportif. Les participants, mais aussi par instants les arbitres, adoptent une gestuelle inspirée des boxeurs, sautillant sur place avec les poings en avant. Pendant son tour de parole, le rappeur adopte généralement une attitude de défi, s'approchant de son adversaire pour le toiser avant de se reculer pour le montrer du doigt d'un air méprisant, prenant à parti le public. Sans jamais recourir à la moindre violence physique, qui témoignerait d'une perte de contrôle et d'une sortie des règles du jeu, le concurrent doit détruire, réduire à néant son adversaire. La proclamation du vainqueur évoque toutefois celle d'un champion sportif, et reste toujours ici entourée d'une atmosphère de fair-play. Il arrive souvent que le perdant félicite son adversaire, le sourire aux lèvres; certains participants, tel Gaïden, adoptent même une attitude amicale de ce type pendant le match même, acquiesçant et riant à certaines "vannes » de leur rival. Le rappeur doit faire montre d'un contrôle absolu de tous les aspects de sa performance, en particulier de sa performance verbale. S'il décide de mimer la colère et l'agressivité, comme le fait Lunik lors de son battle contre Alpha Wann, cela doit être une colère et une agressivité stylisées. Dans ce cas, Lunik lance ses rimes riches en allusions pornographiques de manière brutale, mais sa voix bien qu'agressive est posée avec minutie, avec des effets de rythme très maîtrisés. L'élément le plus directement rédhibitoire est le mutisme, le «blocage », qui contredit tous les présupposés de la performance de rap - prestance, présence, maîtrise, humour et détachement. Parmi les six battles, quatre sont perdus par l'un de leurs concurrents en raison de silences de ce type: Alpha Wann échoue contre Lunik, Srin Po contre Gaïden, Logik Konstantine contre Nekfeu et Pen contre Donny S. Pour les deux autres (Blackapar qui perd contre Deen Burbigo et TZN qui est défait par Eff Gee), la décision est plus délicate, et nécessite davantage la prise en compte de critères poétiques précis par les jurés.

12 Ce match de boxe verbal exécuté dans une ambiance survoltée est lui-même un rapport de force, et ses participants thématisent en permanence cette dimension du jeu. Presque toutes les répliques qu'ils profèrent pourraient être reformulées, à différents niveaux, en : « Je suis supérieur à toi, je suis en train de te battre » ou « Tu es inférieur à moi, tu es en train d'être battu par moi ». Les formules du type "Je t'écrase ", "Je t'explose » ou encore «Je te défonce " alternent avec des métaphores comme "J’te mange » (Gaïden, $3: 07)$. Les répliques les plus appréciées par le public sont celles qui expriment ce rapport d'agression au travers d'un jeu astucieux sur les mots qui souligne l'imagination et la maîtrise du rappeur; par exemple, Nekfeu lance à Logik Konstantine pour souligner l'échec cuisant de sa carrière musicale : "Faudrait qu'j'te casse un bras pour qu'tu fasses une radio » $(4: 08)$, jouant sur les deux sens possibles musical ou médical - de l'expression « faire une radio ». Le rapport de force se joue à la fois dans l'image évoquée et dans la forme qu'elle adopte. Le plus souvent, ce rapport est évoqué à travers un ensemble d'images relevant de la pornographie. Ainsi, dans une certaine mesure, le recours à un imaginaire du rapport sexuel hétérosexuel ou, le plus souvent, homosexuel sert de métaphore au combat verbal lui-même. Au-delà de la transgression, qui appartient certes en plein aux codes du hip-hop, l'image pornographique est le symbole d'un rapport d'agression et de pouvoir. Outre la citation de Gaïden que nous reproduisons dans le titre de cet article, ou celle de TZN ordonnant à Eff Gee : « Mets-toi à quat'pattes ce soir j'vais t'violer » $(2: 24)$, on peut penser à Alpha Wann lançant à propos de Lunik : "Je baise cette bitch » $(2: 34)$, à Logik Konstantine disant à Nekfeu : « Moi j'te baise » $(3: 01)$, ou encore à Eff Gee assurant à TZN : "J'te ken mec » $(3: 07)$. Le rapport sexuel hétérosexuel comme homosexuel est toujours utilisé 
pour figurer un rapport de domination brutale. Le concurrent ou ses proches féminines sont présentés comme les dominés au sein de cette relation, en des termes évoquant l'humiliation. L'imaginaire des films pornographiques hardcore est tout particulièrement utilisé pour mettre en scène des pratiques sexuelles perçues comme humiliantes : "J'urine sur ta meuf, avec neuf reufs on la fuck dans une teuf et j'fous ça sur un dvd d'cul » (Alpha Wann, 9:54), "J'éjacule sur tes sourcils» (Gaïden, 2:21), «J'parle pas sur sa maman donc j'dirai pas qu'j'la baise / ni que quand j'la nique j'lui écrase la tête » (Nekfeu, $5: 57$ ).

13 Cet imaginaire sexuel brutal fonctionne comme une métaphore de la joute verbale, qui elle-même rejoue les rapports de domination et d'agression entendus au sens général. L'univers du hip-hop porte de fait en lui une vision extrêmement brutale et nihiliste des rapports sociaux. Le rap français s'est emparé de cette éthique de la survie et de l'individualisme. De nombreux rappeurs ont pu, à titre personnel, élaborer sur disque des univers plus positifs ou politiquement engagés, tel MC Solaar, Disiz la Peste ou Rocé ; mais dans le cadre de battles, l'imaginaire le plus street, c'est-à-dire le plus authentique, se doit de prévaloir. D'ailleurs même Gaïden, qui participe au Rap Contenders, propose lors de ses performances individuelles en tant qu'artiste des textes d'un tout autre type que ceux qu'il déclame lors du tournoi, n'hésitant pas à traiter de thèmes sociaux et politiques. Organiser un battle, c'est toujours revenir à une sorte d'état premier du hip-hop, état en partie mythique, le back in the days, l'âge d'avant la commercialisation. Cette construction par les acteurs du mouvement hip-hop de l'histoire de ce dernier ne correspond qu'en partie à sa véritable genèse, mais n'en est pas moins extrêmement prégnante dans leurs discours. Pour le dire comme Dyson (2004, 427), « On voit déjà s'ériger autour du rap un mur de mythes qui exclut certains traits cruciaux de l'histoire du hip-hop lui-même. $»^{4}$ Il y aurait d'une part, le hip-hop tel que nous le connaissons au quotidien, comme genre musical diversifié et prisé par la jeunesse de toutes les classes sociales, diffusé sur les chaînes musicales comme sur Internet et commercialisé par la grande distribution, et d'autre part, un hip-hop «à l'état pur ", étranger à toute compromission comme à l'élaboration d'univers musicaux hybrides et singuliers, dans lequel régnerait la rugosité de la rue et la transmission de codes et de valeurs légués par les " anciens", inventeurs du rap old school. Le battle se présente comme une cérémonie réactualisant cette vision puriste du hip-hop, quand bien même elle impliquerait directement des vedettes de la musique grand public, comme c'est le cas par exemple du tournoi Ultimate Clashers avec le groupe Sexion d'Assaut. Cette configuration explique en grande partie le contenu ordurier des couplets proférés par les rappeurs. Alors que dans le rap auquel nous sommes confrontés au sein de l'industrie musicale, de nombreux artistes mettent en avant des textes au contenu varié et souvent engagé politiquement, tels Casey, Despo Rutti ou Oxmo Puccino, le contenu des paroles de rap de battle s'avère extrêmement monotone. Nulle perspective politique n'est défendue, pas plus que des récits de vie émouvants ou édifiants ne sont proposés comme c'est le cas dans le hip-hop au sens plus général. Seules sont échangées des rimes assassines, réduisant le contenu des couplets à un but d'agression.

On peut donc dire en résumé que les éléments de transgression, tels l'usage d'un vocabulaire et d'une imagerie pornographiques et scatologiques, sont mis au service d'une agression symbolique de l'autre. Ce qui fait la force du battle en tant que rite poétique collectif procède de ce paradoxe : c'est au travers de ce par quoi les joueurs sont réunis, de ce par quoi ils se reconnaissent comme pairs, qu'ils s'entredéchirent. 
Dans ce cadre, l'outrance langagière perd en grande partie sa portée transgressive directe pour devenir un protocole ludique et identitaire. La transgression en tant que telle a été revendiquée au sein du hip-hop: le logo Parental Advisory: Explicit Lyrics "Avertissement parental: paroles explicites" imposé en 1985 par la Recording Industry Association of America a par exemple été détourné dans de très nombreux produits dérivés prisés par les passionnés de rap, comme des tee-shirts ou des affiches. L'image sulfureuse de provocateurs bravant les tabous a indubitablement joué un rôle central dans la popularisation de cette culture urbaine. Mais dans le battle, la transgression verbale est si constante et presque mécanique qu'elle n'est plus un but en soi, mais sert de moyen pour s'imposer et détruire l'autre symboliquement. Il s'agit de le pousser hors de scène, de le rendre si ridicule qu'il en perdra toute crédibilité aux yeux du public. L'utilisation d'injures à caractère scatologique et sexuel, à l'évidence, constitue un moyen sûr et efficace de se rallier le public et les juges. Dans un univers exclusivement masculin et survolté, les plaisanteries salaces offrent l'assurance d'une certaine efficacité pragmatique. On peut s'en convaincre en observant les battles de notre corpus. Alpha Wann par exemple, en difficulté contre Lunik, lance au public en mimant une fellation: "Devine c'qu'y s'met dans l'œsophage » $(2: 18)$. Cette réplique ne répond guère à des critères très exigeants d'inventivité verbale, et elle peut apparaître comme relativement paresseuse. Les rimes précédant cette punchline sont en [o] et le mot en [aj] qui a été prononcé, « fromage », l'a été trop longtemps auparavant : il n'est plus assez présent dans la mémoire auditive du public pour que l'effet de reprise sonore soit efficace. En outre, l'usage du mot rare "œsophage ", qui pourrait paraître original dans le rap - et donc donner de la valeur à la réplique - semble au contraire trahir un manque d'inspiration. En effet, le rappeur Booba a dans une chanson de 2004 intitulée "Hors-Saison" attaqué ses ennemis en sous-entendant qu'ils avaient des rapports homosexuels oraux, dans des termes similaires : «Leur technique de dopage laisse des traces de sperme de toutes les ethnies dans l'œsophage ». Alpha Wann ne fait pas explicitement le choix de l'hommage ni du clin d'œil, ce qui fait de cette vanne un élément assez faible en termes de créativité et d'originalité. Les juges ne s'y trompent d'ailleurs pas, qui donnent la victoire à Lunik. Lors de la joute, pourtant, l'effet de cette réplique pornographique est immédiat et l'on entend dans le public des rires fournis. La réplique mettant en scène l'adversaire dans un rapport homosexuel, même si elle est peu inventive et ne comporte pas ou peu d'effets sonores ou rythmiques, fait rire le public en raison de son seul contenu. Le rapport sexuel redouble le combat verbal pour mettre en scène des rapports de force brutaux symboliquement situés sur le terrain du "quartier ", dont les membres du public et le jury sont les dépositaires. Les termes et les images transgressifs sont employés pour mettre à mort l'adversaire, détruisant le personnage dont il s'est doté sur la scène du hip-hop; il faut le faire quitter symboliquement cette scène, l'expulser hors du jeu et de la communauté : «J'sais pas quand t'as commencé l'rap, mais j'sais qu'ça s'termine dans six minutes ", lance Eff Gee à son adversaire (2: 45). Les valeurs de virilité, de fierté, de courage, de maîtrise et de distance, concrétisées dans l'image du sujet singulier triomphant de l'adversité grâce à son talent et à sa persévérance, se manifestent ici de façon spectaculaire dans un flot d'injures ordurières et de vantardise hyperbolique. 


\section{Frapper « sous la ceinture » pour « frapper juste »}

15 Guerre des images, le battle l'est de manière très concrète et aisément observable. Le jeu se situe bel et bien au niveau de l'imaginaire, et en particulier de celui du public. Traiter l'autre de "sale homo" ou de "pédé» comme dans le cadre d'injures homophobes non liées à un jeu poétique ne suffit pas: il faut avoir recours à une imagerie pornographique qui stimule les imaginations et provoque l'hilarité. La transgression langagière, qui donne du relief à une réplique, est ainsi au service de l'efficacité rituelle. Au travers de sa maîtrise des mots, le clasheur se doit de transformer l'image que le public se fait de son adversaire. Son discours est orienté vers la destruction de l'autre comme persona constituée au sein du hip-hop. En cela, le battle se nourrit bien du fonctionnement pragmatique de l'injure, et en particulier de l'injure spécifique, qui cherche un certain nombre de qualités dans l'injurié ou dans l'image que le public se fait de ce dernier. Comme l'a montré Evelyne Larguèche (1983, 25), l'injure est

bien plus que le qualificatif, la transformation du positif en négatif. [C'est une] transformation montrant ce qui est caché, révélant ce qui ne se voit pas, véritable retournement du côté face vers le côté pile, du devant vers le dos, et ce, dirionsnous, en un «tournelangue» (comme on manie un objet habilement et promptement en un tournemain).

16 L'usage des termes et images relevant d'imaginaires pornographiques et scatologiques porte ainsi des coups symboliques à l'image de l'autre, tout en démontrant en retour la maîtrise du locuteur. Il s'agit de faire voir son adversaire sous un autre jour, de mettre en miettes tous les éventuels éléments positifs attachés à sa personne et à sa persona.

C'est à ce niveau précisément que l'axiologie complexe attachée aux mots et images pornographiques et l'efficacité pragmatique se rejoignent. Le rappeur, pour triompher au sein d'un battle, doit effectuer de manière en partie inconsciente une série de choix discursifs qui engagent le positionnement de son adversaire ainsi que le sien propre au sein d'un système de valeurs qu'il estime être celui de son audience-juge. Son travail relève en grande partie de la conjecture, et engage son interprétation de ce qu'est la " communauté hip-hop» et du rapport des membres du public à celle-ci. Ce travail requiert une part d'observation et peut amener à l'invention de répliques originales qui témoignent d'une forme de virtuosité verbale ; bien souvent, cependant, les rappeurs se contentent de se reposer sur ce que nous pourrions nommer des "valeurs sûres ", dans les deux sens du mot «valeur ». En l'absence de trouvailles véritablement inattendues inspirées par l'adversaire et son image, il est toujours peu risqué de se fier aux plaisanteries sexuelles homophobes et misogynes. Évidemment, les valeurs négatives associées à ces éléments ne sont pas propres à l'univers du hip-hop, qui ne fait dans une large mesure que refléter des valeurs présentes dans l'ensemble de la société. Sa seule spécificité a été de transformer ces éléments d'insulte et de moquerie en formules extrêmement codifiées, et dont l'usage est si fréquent qu'il ne surprend en rien l'auditeur averti. Dans d'autres formes d'expression culturelles, il arrive également que des images et des idées transgressives relatives à la sexualité soient transmises, mais la plupart du temps, il s'agit là de moments marqués par un statut relativement exceptionnel. Des films, des romans ou encore des chansons de tous genres peuvent évoquer les rapports charnels hétérosexuels et homosexuels en des termes outrageux, que ce soit par leur dimension explicite ou par leurs implications morales, leur homophobie ou leur misogynie. Mais il s'agit généralement d'épisodes à part entière, 
qui thématisent au moins en partie ces questions. Rien de tel dans le rap de battle: la réplique pornographique transgressive y est tout simplement, pourrait-on dire, une figure.

Pour comprendre cette nuance, nous pouvons comparer le rap de battle qui compose notre corpus à un autre genre musical. Dans la Caraïbe, un certain nombre d'artistes évoluant dans le dancehall proposent des chansons dont le thème même est l'homosexualité, décrite comme une perversion insupportable et un crime. D'une extrême violence, certains morceaux appellent au meurtre des homosexuels. Par exemple dans Boom Bye Bye, le chanteur jamaïcain Buju Banton appelle à assassiner les homosexuels: Me say boom bye bye Inna batty bwoy head " Je dis bang bang bye dans la tête d'un sale pédé ». Cette thématique haineuse n'est pas exceptionnelle et plusieurs centaines de chansons appartenant à ce genre, y compris de véritables tubes radiophoniques, comportent un thème similaire. Le rap, pour sa part, utilise l'homosexualité comme un moyen pour traiter d'autres sujets et fait mine de ne pas l'évoquer en tant que telle. Si nous observons notre corpus, les injures homophobes ne manquent pas : les usages constants des mots "pédé » (Deen Burbigo, $3: 20$; Blackapar, $4: 29$; Lunik, $4: 58$ et $11: 45$ ), « pédale » (Deen Burbigo, $3: 42$ ), « pédéraste » (Lunik, 5 : 24), « tapette » (Gaïden, $5: 56$ ) ou encore « suceur de bites » (Nekfeu, $4: 08$ ) ne laissent guère de doute quant aux valeurs endossées par les rappeurs. Cependant, il n'est jamais directement question de l'homosexualité ni des homosexuels. Les rappeurs utilisent un imaginaire homophobe pour s'en prendre à leur adversaire, mais n'évoquent guère l'homosexualité comme un thème. Dans le rap au sens plus général, les chansons parlant de l'homosexualité sont très rares. Même la chanson du groupe Brand Nubian Pass the Gat, qui a provoqué certaines polémiques pour son homophobie, utilise l'expression Shoot the faggot in the back for actin » like that "J'ai tué ce pédé d'une balle dans le dos pour le punir ", pour décrire un policier, sans que l'usage du terme faggot ne sous-entende que celui-ci était réellement homosexuel.

L'usage transgressif d'images pornographiques, homosexuelles le plus souvent, est ainsi bien une figure, voire une figure imposée. Aucun rappeur dans le tournoi Rap Contenders n'en fait l'économie, fut-ce pendant un seul tour de parole. La misogynie et l'homophobie ne sont pas assumées en tant que postures idéologiques, mais sont sousentendues au sein de nombreuses répliques comme s'il s'agissait de valeurs indiscutables qui cimentent le groupe de pairs. Bien des rappeurs par ailleurs, lors d'entretiens, jurent ne pas être réellement homophobes ou misogynes, mais utiliser ces images parce qu'elles font partie des codes du genre. Par exemple en mars 2009, suite à la polémique provoquée entre autres par son titre "Sale pute ", le rappeur Orelsan a affirmé partout que les propos haineux tenus dans cette chanson ne reflétaient pas ses positions personnelles sur la question des femmes. Dire «salope » ou " pédé » dans le rap est presque aussi automatique que de dire que l'on aime quelqu'un au point de pouvoir en mourir dans un poème romantique, cela ne dit pas nécessairement la vérité de la personne qui assume ce discours, mais témoigne de la force de codes liés à un genre. D'où les vives polémiques aux États-Unis ayant suivi la révélation de l'homosexualité du meilleur ami du rappeur Notorious B.I.G., et plus encore la tolérance dont aurait fait preuve ce dernier vis-à-vis de l'orientation sexuelle de son ami. Comment le rappeur incarnant le plus, dans l'imaginaire des passionnés de rap, les valeurs de virilité et de dureté aurait-il pu accepter l'homosexualité d'un proche? Cet exemple démontre combien l'homophobie, la misogynie, et en particulier leur surgissement au travers d'images transgressives et pornographiques, ont un statut 
ambigu - entre valeurs et éléments de codification poétique. D'une certaine manière, l'homophobie présente dans le hip-hop est plus difficile à dénoncer que celle présente dans les chansons de dancehall que nous citions plus haut. Cette idéologie se nie en effet en tant qu'idéologie, c'est-à-dire en tant que système de valeurs assumé par les locuteurs, pour devenir une sorte de toile de fond rendue visible par ses manifestations indirectes.

$20 \mathrm{Au}$ premier plan de ces valeurs extrêmement traditionnelles endossées sans l'être réellement, le seul thème des battles est la grandeur du locuteur et la petitesse de son adversaire. À partir de ce schéma premier, chaque intervenant élabore des stratégies discursives propres, mais dans lesquelles se manifestent toujours d'une façon ou d'une autre les symboles essentiels - core symbols diraient les anglophones - de la culture commune. Tous les participants sans exception adoptent une posture discursive qui met en avant leur propre virilité. Cette persona se construit tout autant par leurs discours que par leurs postures et leurs choix vestimentaires. Les images de la masculinité convoquées imposent à tous de se tenir très droit, de bomber le torse, ou encore d'adopter des postures de défi par des gestes des mains ou des regards menaçants. Les tenues des rappeurs témoignent également d'une valorisation de la virilité : casquettes sur la tête (Alpha Wann, Blackapar, Dony S, Eff Gee, Nekfeu, Pen, Stunner) ou bonnet (Deen Burbigo, Logik Konstantine, Lunik), chaussures de sport, pantalons larges et pulls à capuche confèrent à tous une apparence vestimentaire socialement marquée comme urbaine (streetwear) et virile. Un participant qui se permettrait de monter sur la scène vêtu d'une manière perçue comme trop sophistiquée ou soignée s'exposerait à des moqueries fournies, lesquelles emprunteraient à coup sûr un lexique homophobe. Les injures, et en particulier les injures à portée sexuelle, visent à tuer la persona que s'est soigneusement confectionnée l'autre participant. Par exemple, Alpha Wann se donne dans son premier tour de parole une image relevant dans cet univers culturel de la masculinité. Il multiplie les injures homophobes $(2: 04,2: 15,2: 34)$ et reprend à son compte les poncifs machistes du gros pénis $(2: 37)$ et de la puissance sexuelle hyperbolique: «Tu ken moins d'bitch que moi » $(3: 01)$. Pour le prendre à défaut, son adversaire Lunik s'empare d'un élément de son habillement qui a attiré son attention afin de le féminiser: "Tu crois qu'c'est un collier autour du cou que t'as? P'tit pédé de bouffon j'crois qu'c'est des boules de geisha » $(4: 58)$. Le collier, qui n'appartient pas à la panoplie traditionnelle du rappeur, est utilisé par Lunik pour détruire les éléments de masculinité dont Alpha Wann avait tenté d'orner sa propre persona lors de son tour de parole. Or, on le voit, la transgression langagière est dans ce cas appelée en renfort pour donner de la force à cette punchline, à travers la référence au jouet sexuel féminin que sont les boules de geisha.

21 Nous sommes en présence d'une guerre des mots dans laquelle chacun se met en scène sous les traits d'un "vrai mec », d'un "bonhomme ", recourant en particulier à des personnages centraux de l'imagerie du rap américain comme le gangsta " gangster » et le pimp «maquereau ». Ces personnages symbolisent un ensemble de valeurs conçues comme emblématiques du mâle hétérosexuel, et sont inséparables des mythologies du hip-hop dont nous parlions précédemment. Pour Kalamka et West $(2006,198)$, celles-ci relèvent en effet de constructions a posteriori qui procèdent par tri et par effacement, guidées par un idéal de pureté - notamment sur le plan sexuel : «Cette mythologie (...) s'enracine dans une réticence à explorer la manière dont des sexualités diverses ont historiquement fait partie de la culture hip-hop. $»^{5}$ Comme nous l'avons vu avec Alpha 
Wann, la sexualité sert à affirmer symboliquement sa propre virilité, même si cela est parfois fait avec une certaine dose d'autodérision. Le rappeur qui a la parole ne cesse d'affirmer qu'il est un homme à la virilité éclatante et agressive, et que son adversaire endosse un rôle féminin. Cette répartition des rôles confère de manière apparemment paradoxale aux battles une tonalité homosexuelle évidente. En effet, pour se présenter comme un parangon de la virilité, le locuteur se met en scène dans des rapports sexuels, tantôt avec une femme proche de son adversaire (mère, sœur, petite amie), mais aussi et surtout avec l'adversaire lui-même. Parfois, la représentation de ce rapport de force stylisé sous la forme d'une relation charnelle homosexuelle opère un certain nombre de détours qui permettent d'éviter le tabou. Deen Burbigo lance par exemple à Blackapar: "Moi mes rimes agressives t'laissent comme une péripatéticienne d'vant les bites athlétiques mec» $(10: 21)$. Ici, la virilité de Deen Burbigo est soulignée par l'adjectif «agressives", qui qualifie ses rimes. Leur aspect percutant est du reste souligné par le jeu phonique qui joue par répétition d'une série de six sons vocaliques - [e], [i], [a], [e], [i] et [ $\varepsilon$ ]. La comparaison en " comme » établit une analogie avec un rapport sexuel monnayé, au sein duquel l'adversaire serait une prostituée et le locuteur un client évidemment doté d'une «bite athlétique ». Dans d'autres cas le rapport sexuel est décrit directement. Pour ne citer que Nekfeu lors de son battle contre Logik Konstantine : «Quand je t'emmène en ballade t'as mon sexe au cul » $(2: 32)$, «Et si tu m'vois m'ronger les ongles c'est pour pas m'faire mal quand j'te doigte » $(2: 42)$, «La nuit j'me défoule contre tes textes [il tape deux fois dans ses mains] c'est le bruit de mes boules contre tes fesses» $(7: 11)$, «J'attrape tes sales tresses puis te redresse pour te claquer les fesses / Tu n'peux pas m'baiser comme si j'avais mes règles, salope » $(7: 46)$. Notons également que cette stylisation sexuelle du rapport de force viril emprunte souvent des accents raciaux. En effet, dans l'univers du hip-hop, certains stéréotypes aujourd'hui souvent considérés comme racistes ont été repris par les rappeurs dans un sens valorisant. C'est le cas en particulier de l'association des hommes noirs avec une virilité exacerbée. Dans le rap américain, se décrire comme un "vrai mec" passe souvent par l'emploi d'expressions racialement marquées comme real nigga "vrai négro ». Ce type de provocation à la fois sexuelle et raciale n'est pas absente du rap français. Dans sa chanson de 2006 intitulée « Le Duc de Boulogne ", Booba dit par exemple que son rap « est pour les enfants terribles qui ne font aucun effort / $\mathrm{Au}$ parcours comme une queue d'négro, long et pénible ». Cet imaginaire qui témoigne de ce que les féministes américaines comme Kimberlé Crenshaw $(1998,358)$ ont pu nommer l'intersectionnalité (intersectionality), c'est-à-dire l'étroite interdépendance entre les catégories de genre et les catégories raciales, est également présent dans notre corpus. Lors du battle entre Alpha Wann et Lunik - tous deux d'origine africaine - ce dernier lance par exemple à son adversaire : «Les Noirs en ont un gros t'es l'exception qui confirme la règle » $(5: 09)$. Puis, afin d'achever cette entreprise de féminisation - donc de dénigrement - Lunik ajoute, mettant en tension ces images conçues comme mutuellement exclusives par la pensée essentialiste que sont la « négritude » et l'homosexualité : « C'est l'genre de Noir qui porte des couches et boit des coups dans l'marais : c'est un putain d'pédéraste» $(5: 24)$. L'image, parmi d'autres du même type, provoque les rires du public et permet à Lunik de gagner ce combat. L'injure vaut pour son efficacité, pour ses effets les plus directs: la transgression se fonde sur les éléments positifs mis en avant par le locuteur adverse pour les cibler en priorité, déconstruisant sa carapace de valeurs à grands coups de 
mots interdits. Cet effet de mise en circuit de la transgression verbale amène à une sorte d'hyper-visibilité paradoxale des interdits.

\section{Les paradoxes de l'obscène}

22 La spontanéité, nous l'avons vu, constitue l'un des éléments centraux de l'imaginaire du battle. Nous nous trouvons dans un rituel qui, malgré sa dimension en partie préparée, emprunte les apparences du déchaînement immédiat et non maîtrisé. Cet élément d'ordre rituel pourrait paraître sans rapport avec les valeurs associées à la sexualité que nous venons d'évoquer, mais il n'en est rien. La mobilisation obsessionnelle de valeurs traditionnelles en toile de fond d'un affrontement symbolique entre pairs traduit une étroite association entre cet élément poétique et l'axiologie qui le soustend. La double transgression des tabous langagiers et des tabous moraux associés à l'homophobie et à la misogynie devient l'outil d'une lutte pour la maîtrise de codes communs, et non une fin en soi ; mais le combat simulé ne suffit pas à cacher le fait qu'à un autre niveau de lecture, les participants exaltent indirectement un même ensemble de codes et de valeurs. Pour le dire comme Bourdieu (1992, 279), «la lutte pour le monopole de la légitimité contribue au renforcement de la légitimité au nom de laquelle elle est menée ». Ici, les éléments ayant trait au déroulement du rituel poétique sont indissociables d'une praxis de l'authenticité, qui amène sur le plan des usages des éléments sexuels à un double paradoxe.

Derrière les mots et les gestes du rituel poétique du battle, se dessine en creux une pensée du monde hantée par l'idée de fausseté. La part d'acquis que comporte la cérémonie poétique est passée sous silence afin de rendre possible l'étroite association entre masculinité et authenticité qui sert de moteur à la compétition. À travers des traits d'esprit, des rimes et des effets de citation et de parodie - en somme, à travers des effets - il s'agit toujours de revendiquer un statut d'authenticité. Les rimes "viennent des tripes", elles ne sont jamais copiées sur d'autres rappeurs. Nous pourrions citer ici la judicieuse formule de Deborah Root $(1996,78)$ selon laquelle "l'authenticité est la monnaie en vigueur sur le marché de la différence culturelle » (authenticity is the currency at play in the marketplace of cultural difference). Le hip-hop, même s'il est né dans les années 1970 dans le Bronx, ne peut se comprendre sans une prise en compte du contexte bien plus vaste au sein duquel il est devenu le phénomène culturel global que nous connaissons, et au sein duquel les participants du Rap Contenders, nés dans les années 1970 et 1980, ont grandi. Le rap a été popularisé par des radios, des télévisions, des maisons de disques ou encore des sites Internet qui l'ont fait passer d'un phénomène local à un genre musical consommé par des personnes de tous les pays et de toutes les classes sociales. Si les acteurs du mouvement affichent le plus souvent une identité liée d'une part aux ghettos urbains et aux classes sociales défavorisées, d'autre part aux personnes noires et aux minorités ethniques en général, leur audience est infiniment plus vaste. L'immense majorité du public du rap est composé de personnes blanches, et les passionnés de ce genre comptent parmi leurs rangs des personnes d'origine très aisée, ainsi que des femmes et des membres des communautés lesbienne, gay, bisexuelle et transsexuelle (LGBT). Comme l'a montré Imani Perry $(2004,136)$, c'est dans une très large mesure cet iconoclasme du rap par rapport aux normes exprimées au sein de la société dans son ensemble qui l'a rendu si attirant. Sur le plan des rapports entre les genres, l'affirmation d'une hétérosexualité 
agressive a pu attirer un public masculin nombreux, en ces temps d'acceptation progressive - quoiqu'incomplète - des arguments défendus par les mouvements féministes et LGBT. Le rap, jusque dans sa diction, se présente comme le son authentique de "la rue ", de la "vie véritable ", et c'est en partie cela qui fait sa valeur sur le marché des musiques urbaines ou exotiques. En outre, il affirme au travers de cette rhétorique de l'authenticité des images identitaires rassurantes, en particulier sur le plan des genres. Pour des générations de jeunes hommes de tous milieux et de toutes origines, cette pratique culturelle a pu permettre de soulager une anxiété liée à la quête d'une image de soi en tant que mâle hétérosexuel. C'est peu ou prou en ces termes que Kantor $(2009,145)$ s'explique la persistance de discours homophobes agressifs du type de ceux que présente notre corpus urbain :

Certains hommes homophobes n'ont jamais réussi à dépasser leur lutte adolescente pour développer et maintenir une identité masculine, même si l'idéal masculin sur lequel se fondait cette identité était simpliste. Ces hommes s'en prennent émotionnellement ou physiquement aux homosexuels (...) pour développer une image machiste d'eux-mêmes dont ils puissent se sentir fiers ${ }^{6}$.

La rhétorique de l'authenticité permet de couper court à toute discussion rationnelle pour faire retour à une forme de "raisonnement par l'émotion » (emotional reasoning, Kantor, 2009, 132), de "penser-ressentir» (feelthink, ibid., 4). Le couplet de rap se présente comme provenant des tréfonds de la rue, des tréfonds du réel : le $\mathrm{MC}$ « baves du béton, crache du béton, chie du béton ", disait Akhenaton dans la chanson du groupe I AM «Demain c'est loin ». Le recours à la transgression langagière et à la représentation également transgressive de scènes sexuelles est au service de la mythologie du hip-hop, qui pose à son tour une série de tabous qui l'empêchent, pour emprunter ses mots à Didier Éribon $(2008,141)$, de pouvoir « accueillir la multiplicité infinie des choix individuels et des modes de vie ».

Nous sommes en présence d'un rituel poétique ludique qui mobilise un certain nombre de symboles et de valeurs pour permettre à ses participants de jouer ensemble à un même jeu. Le degré d'adhésion de chacun à ces symboles et à ces valeurs demeure très variable, et impossible à vérifier de manière certaine. Pour reprendre l'exemple que nous prenions plus haut, rien ne permet d'affirmer qu'un poète d'inspiration romantique - jurant dans ses poèmes qu'il aime une femme à en mourir - ressente réellement ces sentiments ou qu'il agisse en conséquence dans le cadre de sa vie privée. L'invention poétique mobilise des valeurs, mais sans nécessairement abolir toute distance subjective avec elles. Le rap invite ses locuteurs à se fondre dans une persona poétique largement construite sur le modèle du groupe de pairs des ghettos urbains. Imani Perry $(2004,133)$ explique à ce titre :

Les relations entre hommes dans le hip-hop, à la fois hostiles et chaleureuses, ont souvent été vues comme des manifestations d'une hyper-masculinité. Le fait que les relations entre pairs se fassent presque toujours entre hommes et non par-delà la barrière du genre est souvent décrit comme un signe de sexisme ${ }^{7}$.

Le féminin est effacé, exclu de fait de cet univers dans ses manifestations se présentant comme les plus « authentiques». Keyes $(2002,135)$ cite à ce propos Nathan McCall, un homme qui a grandi dans la rue et connaît bien son univers :

Dans la sagesse de la rue, il y a deux sortes de femmes: il y a les femmes, comme votre mère, votre sœur ou votre prof, et il y a les salopes et les putes. Les salopes et les putes ne sont bonnes qu'à une seule chose - baiser ${ }^{8}$. 

Lorsqu'un locuteur souhaite se comparer à une personnalité reconnue, il fait toujours le choix de personnalités de sexe masculin : par exemple les rappeurs Booba et Rohff (Deen Burbigo, 9:45) ou Tupac et Biggie (Pen, 1:51), ou encore les sportifs Shaquille O'Neal (Eff Gee, $3: 03$ ) et Hussein Bolt (Eff Gee, $5: 46$ ). Il est intéressant de noter que les personnes de sexe féminin évoquées le sont toujours de manière anonyme. Non seulement les femmes ne participent pas au battle, mais elles ne sont jamais nommées en tant qu'individus : il ne s'agit toujours que de «ta mère ", « ta sœur » ou « ta meuf », c'est-à-dire de femmes désignées par leur rapport à l'adversaire masculin. La femme est ainsi effacée, et n'existe plus que comme menace d'une contamination du mâle par un principe féminin facteur d'inauthenticité. La meilleure preuve qu'il s'agit ici de se référer à une idée de la femme en général est l'indistinction totale entre les rapports maternel, fraternel et amoureux. Mère, sœur et petite amie sont au fond la même chose, à savoir des incarnations de la féminité que le mâle dominant se doit de contenir. La femme, et en particulier son désir, sont des menaces à la maîtrise de l'homme sur sa propre vie.

Cette idée se vérifie totalement dans les battles du Rap Contenders, où toutes les évocations de proches féminines de l'adversaire mettent en scène ces dernières dans le cadre de rapports sexuels ou affirment leur propension à s'y adonner de manière incontrôlable. On citera Blackapar: "Mon son tourne dans toutes les caisses un peu comme ta meuf poto » (11); Alpha Wann : «Qu'est-ce tu vas faire à part dire à ta mère qu'elle a oublié son slip chez moi » $(2: 55)$, «J'lâche une info ya... qu'avec toi qu'ta meuf n'est pas une nymphomane » $(6: 14)$; Lunik: «Sa mère est une traînée et c'est d'tous les pénis du quartier que son pubis pue / Elle aurait été blanche elle aurait pris des 
couleurs à force de s'faire tourner dans tous les sens comme un Rubik's cube » $(7: 30)$; «Ta chienne de mère et ta sœur sont les groupies que t'as jamais eues / Pendant qu'sur scène tu brailles devant trois spectateurs elles s'font tourner en coulisse par la sécu " ( $8: 17)$; Gaïden: "Ta meuf est chaude comme un curé sous la soutane» (7:23); Nekfeu: "T'as pas un centime ta femme l'a senti j'suis gentil j'la fais kiffer comme l'alarme incendie » $(2: 20)$, "Je passe chez ta tasse et lui rentre cash dans la chatte " ( $4: 24)$; et enfin Eff Gee : "Ta bitch t'a quitté, j'la ken avec brutalité » $(8: 19)$. Ce type d'injures à la mère, la sœur ou la petite amie sont plus valorisées encore lorsqu'elles s'accompagnent d'effets stylistiques originaux par exemple un jeu sur la polysémie d'un mot. C'est ce que fait Deen Burbigo lorsqu'il dit à propos de sa propre rime : «J'avoue qu'celle-là elle était facile un peu comme la meuf de black pute» (6:36). La maîtrise stylistique se doit d'accompagner une mise en scène de soi comme mâle dominant qui maitrise les femmes qui l'entourent, à l'inverse de l'adversaire dont les mère, sœur et petite amie sont des délurées incontrôlables qui sèment le trouble - le trouble dans le genre, dirait Judith Butler.

Les représentations du rapport homosexuel participent de la même intrication entre rituel poétique et valeurs et aboutissent elles aussi à un paradoxe, qui peut nous amener à refermer cette étude par une interprétation du rôle de la transgression langagière ici. Ces locuteurs se présentent de manière obsessionnelle comme des hétérosexuels, c'est-à-dire comme des hommes aimant les femmes, mais effacent littéralement les femmes du rituel poétique. Par ailleurs, ils prétendent abhorrer l'homosexualité, qui incarne la fausseté et l'inauthenticité par excellence, et passent le plus clair de leurs couplets à se mettre en scène avec leur adversaire du même sexe dans les postures sexuelles les plus explicites. L'acte sexuel avec la femme est valorisé, mais celle-ci est effacée par un détour à un imaginaire de la domination, du contrôle. Le rapport homosexuel est dévalorisé, mais envahit les discours de façon troublante. On peut ainsi poser l'hypothèse selon laquelle la transgression langagière, qui passe par l'évocation d'éléments sexuels explicites de manière inappropriée aux yeux de la société, sert en dernier lieu à une reprise en main symbolique. Pour le dire autrement, la transgression finit par servir l'exact contraire d'une transgression. La possibilité même du sujet féminin et de son désir, source d'inquiétude, est balayée par le recours aux images pornographiques outrancières. Au-delà des femmes en tant que telles, c'est bien le féminin qui est mis sous tutelle, compris comme un principe d'ambiguïté qui viendrait menacer l'intégrité du sujet masculin "authentique». L'homosexualité comme possibilité d'une ambiguïté dans le genre est ainsi mobilisée de manière presque obsessionnelle, pour mieux être reprise en main de la même manière. L'évocation transgressive des rapports sexuels avec des femmes ou entre hommes n'est au final une transgression que sur le plan des imaginaires linguistiques et culturels. Lorsque nous observons le rituel poétique ludique et la manière dont il utilise ces évocations, cette transgression apparente se révèle au contraire comme le signe d'une quête de permanence, de stabilité rassurante, dans un monde où les certitudes sur les identités sont de plus en plus remises en question. Dans cet art du «viol » verbal, la transgression par l'obscène constitue peut-être en définitive un rappel à l'ordre. 


\section{BIBLIOGRAPHIE}

ABRAHAMS, Roger D., 1970, Deep Down in the Jungle. Negro Narrative Folklore from the Streets of Philadelphia, Chicago, Aldine Publishing Company.

BAZIN, Hugues, 1995, La Culture hip-hop, Paris, Desclée de Brouwer.

BÉTHUNE, Christian, 2003, Le Rap. Une esthétique hors-la-loi, Paris, Éditions Autrement.

BOURDIEU, Pierre, 1992, Les Règles de l'art, Paris, Seuil.

CAMPBELL, Kermit E., 2005, Getting our Groove on. Rhetoric, Language and Literacy for the Hip-Hop Generation, Detroit, Wayne State University Press.

CRENSHAW, Kimberlé, 1998, “A Black Feminist Critique of Antidiscrimination Law and Politics" in KAIRYS, David (ed.), The Politics of Law. A Progressive Critique, New York, Basic Books, p. 356-380.

DYSON, Michael Eric, 2004, The Culture of Hip-Hop, in Michael Eric Dyson Reader, New York, Basic Civitas Books, pp. 401-410.

ÉRIBON, Didier, 2008, Contre l'égalité et autres chroniques, Paris, Éditions Cartouche.

HOLT, DeVone, 2003, Hip-Hop Slop. The Impact of a Dysfunctional Culture, Louisville, Milton Publishing.

It's All One. A Conversation between Juba Kalamka and Tim'm West, in cHANG, Jeff (ed.), 2006, Total Chaos. The Art and Aesthetics of Hip-Hop, New York, Basic Civitas Books, pp. 198-208.

KANTOR, Martin, 2009, Homophobia. The State of Sexual Bigotry Today, Westport et Londres, Praeger. KEYES, Cheryl L., 2002, Rap Music and Street Consciousness, Urbana et Chicago, University of Illinois Press.

LARGUÈCHE, Évelyne, 1983, L'Effet-injure. De la pragmatique à la psychanalyse. Voix nouvelles en psychanalyse, Paris, Presses universitaires de France.

PERRY, Imani, 2004, Prophets of the Hood. Politics and Poetics in Hip Hop, Durham and London, Duke University Press.

PINN, Anthony B., 1997, 'Gettin' Grown. Notes on Gangsta Rap Music and Notions of Manhood, Journal of African American Men, 2, 1-4, pp. 61-73.

ROOT, Deborah, 1996, Cannibal Culture. Art, Appropriation and the Commodification of Difference, Boulder, Colorado, Westview Press.

ROSE, Tricia, 1994, Black Noise. Rap Music and Black Culture in Contemporary America, Hanover and London, Wesleyan University Press.

SMITHERMAN, Geneva, 1977, Talkin and Testifyin. The Language of Black America, Boston, Houghton Mifflin Company.

VETTORATO, Cyril, 2008, Un monde où l'on clashe. La Joute verbale d'insultes dans la culture de rue, Paris, Éditions des Archives contemporaines. 


\section{NOTES}

1. Nous avons préféré ici ce terme à celui d'« afro-américain ", conformément aux évolutions des usages aux États-Unis et dans la recherche anglophone.

2. Répliques humoristiques percutantes qui ridiculisent l'adversaire et démontrent la virtuosité verbale du rappeur.

3. "Rooted in the black cultural experience, the semantics of which depends not only on the immediate linguistic context but on the sociohistorical context as well." Toutes les traductions sont de l'auteur.

4. "There is already growing up around rap a wall of myths that excludes crucial features of hip hop's own history."

5. "The mythology (...) is rooted in the reluctance to explore the ways that diverse sexualities have historically been part of hip-hop culture."

6. "Some homophobic men have never gotten over their adolescent struggle to develop and maintain a masculine identity, however simplistic the masculine ideal at the core of such an identity might be. These men emotionally or physically beat up on gay men (...) as part of their own attempt to develop a macho self-image of which they can feel proud."

7. "Relationships between men in hip hop, both hostile and warm, have often been seen as manifestations of hypermasculinity. That loving relationships between peers overwhelmingly appear between men and not across gender lines is frequently described as an expression of sexism."

8. "According to street wisdom, there were two types of females: There were women, such as your mother, sister and teacher, and there were bitches and hoes. Bitches and hoes were good for one thing-boning."

9. "Male sexist narratives often involve devaluing and dominating black female sexuality and sexual behaviour."

10. "To give positive attention to women results in the loss of one's masculine identity. Women must be controlled through language (reduced to hoes, bitches, and skeezers) and sex as a weapon of damage, domination, and control."

\section{RÉSUMÉS}

Les joutes verbales d'insultes propres à la pratique du rap, les battles, mettent face à face deux participants chargés de se détruire mutuellement par les mots. Le langage obscène, et surtout l'évocation très crue de scènes à caractère sexuel, constituent un de leurs ressorts principaux. À partir de l'étude d'un tournoi filmé en 2010 à Paris, nous tenterons de saisir les enjeux de ces transgressions langagières dans les battles de rap. Nous examinerons en particulier les rapports qu'elles entretiennent avec le fonctionnement du rituel poétique, mais aussi avec le système de valeurs qu'il convoque sur un mode ludique.

The form of verbal dueling practiced within the hip-hop cultural community, the freestyle battle, places face-to-face two contestants who are expected to destroy each other through words. Obscene language, and more particularly the very graphic representation of sexual scenes, is one of their most common resources. Beginning with the study of a tournament recorded in 2010 in Paris, we will try to grasp what is at stake in these verbal transgressions. We will examine more 
precisely their relationship to the organization of the poetic ritual, as well as with the value system it brings into play in a ludic way.

\section{INDEX}

Thèmes : anthropologie (Europe)

Mots-clés : rap, joute verbale, injure, sexualité, masculinité, hip-hop, battles (rap)

Keywords : Rap, Verbal Dueling, Insult, Sexuality, Masculinity, Hip Hop Music, Freestyle Battles 